\title{
Association between diet quality indicators and nonalcoholic fatty liver disease: The FLiO study
}

\author{
Cristina Galarregui ${ }^{1}$, M. Angeles Zulet ${ }^{1,2}$, Bertha Araceli Marin-Alejandre ${ }^{1}$, Irene Cantero ${ }^{1}$, \\ Nora Goodwin $^{3}$, J. Ignacio Monreal ${ }^{4,5}$, Mariana Elorz ${ }^{4,6}$, Alberto Benito-Boillos ${ }^{4,6}$, \\ Josep A. Tur ${ }^{2,7}$, J. Alfredo Martínez ${ }^{1,2}$ and Itziar Abete ${ }^{1,2}$ \\ ${ }^{1}$ Department of Nutrition, Food Science and Physiology and Centre for Nutrition Research, Universidad de Navarra, \\ Pamplona, Spain, \\ ${ }^{2}$ Biomedical Research Centre Network in Physiopathology of Obesity and Nutrition (CIBERobn), Instituto de Salud \\ Carlos III (ISCII), Madrid, Spain, \\ ${ }^{3}$ Dublin Institute of Technology (DIT), Dublin, Ireland, \\ ${ }^{4}$ Navarra Institute for Health Research (IdiSNA), Pamplona, Spain, \\ ${ }^{5}$ Clinical Chemistry Department, University Clinic of Navarra, University of Navarra, Pamplona, Spain, \\ ${ }^{6}$ Radiodiagnostic Department, University Clinic of Navarra, Pamplona, Spain and \\ ${ }^{7}$ Research Group on Community Nutrition and Oxidative Stress, Universitat de les Illes Balears, Palma, Spain
}

\begin{abstract}
Introduction: Dietary components are contributing factors in the development of Nonalcoholic fatty liver disease (NAFLD). The glycaemic index (GI), glycaemic load (GL) and total antioxidant capacity (TAC) have been considered potential dietary tools influencing diet-disease relationships. The aim of this study was to evaluate associations of the dietary GI, GL, TAC and insulin resistance (IR) condition with hepatic fat in NAFLD adults.

Material and methods: 112 overweight/obese adults with NAFLD (age: $50.8 \pm 9$ years old) were included in the trial. Dietary intake was assessed by a validated 137-item food frequency questionnaire (FFQ). Anthropometric, glycemic and lipid profiles, fatty liver quantification by magnetic resonance imaging (MRI) and IR measured by the Homeostatic Model Assessment of IR (HOMA-IR) were assessed at baseline. This study was registered as FLiO: Fatty Liver in Obesity study; NCT03183193.

Results: The median of liver fat content by MRI was $6.4(3.8-10.9)$ in the recruited population. Participants with higher liver fat content showed significantly increased values of glucose, insulin, HbA1c and HOMA-IR than those with lower liver fat content ( $\mathrm{p}<$ 0.05). Correlation analyses revealed relevant positive associations of hepatic fat with $\mathrm{GI}(\mathrm{r}=0.17 ; \mathrm{p}=0.077)$ and $\mathrm{GL}(\mathrm{r}=0.19 ; \mathrm{p}$ $=0.047)$. Also, a negative association between liver fat content and TAC $(r=-0.22 ; p=0.023)$ was found. Linear regression analyses were used to examine the associations of hepatic fat and dietary quality indicators as well as IR adjusted for potential confounders (sex, age and physical activity). The final models showed that HOMA-IR, GI, GL and TAC were able to explain between 22.4 and $22.8 \%(\mathrm{p}<0.001)$ of the variability of liver fat content.
\end{abstract}

Discussion: The pathophysiology of NAFLD is thought to be associated with dietary determinants that contribute to metabolic dysregulation such as IR, ectopic liver fat deposition and hepatic damage. In accordance with other authors, we suggest that monitoring GI, GL and TAC may be useful approaches for the dietary treatment of NAFLD since they are related to hepatic fat. Additionally, it is important to highlight the essential role of IR in NAFLD as a key mediator in the development of NAFLD. Certainly, findings of the present study revealed a significant association of hepatic fat accumulation and IR.

In summary, GI, GL and TAC are potential markers of diet quality, with an impact on susceptible population at hepatic risk.

\section{Acknowledgments}

Government of Navarra (61/2015), CIBERobn, Fundació La Marató de TV3 (201630.10). C.G. is receiving a grant from MECD (FPU17/06330).

\section{Conflict of Interest}

"There is no conflict of interest". 